\title{
Modeling Dynamical Dark Energy
}

\author{
R. Mainini, A.V. Macciò \& S.A. Bonometto \\ Physics Department G. Occhialini, Università degli Studi di Milano-Bicocca, Piazza della Scienza 3, \\ I20126 Milano (Italy) \\ A. Klypin \\ Astronomy Department, New Mexico State University, Box 30001, Department 4500, Las Cruces, NM \\ 88003-0001
}

\begin{abstract}
Cosmological models with different types of Dark Energy are becoming viable alternatives for standard models with the cosmological constant. Yet, such models are more difficult to analyze and to simulate. We present analytical approximations and discuss ways of making simulations for two families of models, which cover a wide range of possibilities and include models with both slow and fast changing ratio $w=p / \rho$. More specifically, we give analytical expressions for the evolution of the matter density parameter $\Omega_{m}(z)$ and the virial density contrast $\Delta_{c}$ at any redshift $z$. The latter is used to identify halos and to find their virial masses. We also provide an approximation for the linear growth factor of linear fluctuations between redshift $z=40$ and $z=0$. This is needed to set the normalization of the spectrum of fluctuations. Finally, we discuss the expected behavior of the halo mass function and its time evolution.
\end{abstract}

Subject headings: methods: analytical, numerical - galaxies: clusters - cosmology: theory - dark energy

\section{Introduction}

Observations of high redshift supernovae (Perlmutter et al. 1999; Riess et al. 1998) as well as the analysis of fluctuations of the cosmic microwave background combined with data on the large-scale structure of galactic distribution (e.g. Balbi et al. 2000; Tegmark, Zaldarriaga, \& Hamilton 2001; Netterfield et al. 2002; Pogosian, Bond, \& Contaldi 2003; Spergel et al 2003) indicate that there is a significant component of smooth energy with large negative pressure, characterized by a parameter $w \equiv p / \rho \lesssim-0.5$. This component is dubbed dark energy (DE). The nature of $\mathrm{DE}$ is open for debate with candidates ranging from a cosmological constant $\Lambda$ to a slowly evolving scalar field $\phi$ to even more exotic physics of extra dimensions (e.g., Dvali, \& Turner 2003)

One of the most appealing ideas for DE is a selfinteracting scalar field, which evolves with time (Ratra \& Peebles 1988; Wetterich 1988). We call this dynamical Dark Energy. The advantage of the dynamical DE models as compared with the $\Lambda$ CDM models is that DE naturally yield an accelerated expansion easing the problem of fine tuning. The observational signatures of dynamical DE should be carefully investigated in order to determine which measures can be used to discriminate $\Lambda \mathrm{CDM}$ from dynamical $\mathrm{DE}$ and among different dynamical DE models. In this paper we focus on the two most popular variants of dynamical DE. Ratra \& Peebles (1988, RP hereafter) studied DE models, which cause a rather slow evolution of $w$. Models based on simple potentials in supergravity (SUGRA) result in faster evolving $w$ (Brax \& Martin 1999, 2000). Together RP and SUGRA potentials cover a large spectrum of evolving $w$. The potentials are written as

$$
V(\phi)=\frac{\Lambda^{4+\alpha}}{\phi^{\alpha}} \quad(R P)
$$




$$
V(\phi)=\frac{\Lambda^{4+\alpha}}{\phi^{\alpha}} \exp \left(4 \pi G \phi^{2}\right)
$$

Here $\Lambda$ is an energy scale, currently set in the range $10^{2}-10^{10} \mathrm{GeV}$, relevant for fundamental interaction physics. The potentials depend also on the exponent $\alpha$. Once the parameters $\Lambda$ and $\alpha$ are assigned, the DE density parameter $\Omega_{D E}$ follows. Here, however we prefer to use $\Lambda$ and $\Omega_{D E}$ as independent parameters.

Dynamical DE has a kinetic and a potential components, reading $\dot{\phi}^{2} / 2$ and $V(\phi)$, respectively. Those factors define the energy density $\rho_{D E}$ and the pressure $p_{D E}$. In general, the ratio of the pressure and the density

$$
w=\frac{p_{D E}}{\rho_{D E}}=\frac{\dot{\phi}^{2} / 2-V(\phi)}{\dot{\phi}^{2} / 2+V(\phi)}
$$

changes with time and is typically negative when the potential $V$ is sufficiently large, as one expects to occur in the recent epoch.

In order to simplify the situation, the dynamical DE is often replaced with models with constant $w \neq-1$. This can be considered as a formal generalization of the equation of state of vacuum energy density for which $w \equiv-1$. These models result in accelerated expansion if $w$ exceeds $\approx-1 / 3$. The main advantage of constant $w$ is to yield models easier to deal with than the dynamical DE. Although finding a physical justification for models with constant $w \neq-1$ is more difficult than for the cosmological constant (see, however, Caldwell 2002), these models are still useful as toy models, allowing one to inspect the effects of an acceleration which is slower than with the vacuum energy.

In this paper we show how complications with the dynamical DE can be overcome if one uses suitable approximations, that we provide. Besides of allowing an easier treatment of the dynamical $\mathrm{DE}$, these expressions also allow us to compare the dynamical DE with the models with constant $w$. One of the results of this comparison is that differences between constant $-w$ and dynamical DE are significant, being comparable with those between $\Lambda \mathrm{CDM}$ and constant $-w$.

The results given in this paper are based on a modified version of the CMBFAST code. The modifications include effects due to the change in the rate of the expansion of the Universe and fluctuations of the scalar field. Although these fluctu- ations rapidly fade, soon after their enter the horizon, their effect on cosmic microwave background anisotropies and polarization is quite significant, while they also cause (smaller) modifications of the transfer function on large scales.

In addition, we also estimate the growth of linear and non-linear fluctuations of non-relativistic matter only. Previously our algorithms were used by Mainini, Macciò, \& Bonometto (2003, MMB03 hereafter).

Making use of these algorithms, in this paper we work out: (i) Analytical approximations of the dependence of the matter density parameter $\Omega_{m}$ on the redshift $z$; (ii) Modifications to run $N$-body simulations of clustering of dynamical DE models; (iii) Analytical approximations for the virial density contrast $\Delta_{c}$ at any redshift $z$. Expressions derived from the linear theory can also be used to compare the observables deduced for dynamical DE and for constant $w$. We argue that these approximations make an analysis of the dynamical $\mathrm{DE}$ as simple as for models with constant $w$.

\section{The virial density contrast}

We start with finding the evolution of the density contrast in the top-hat approximation for models with DE. Considering a spherical fluctuation greatly simplifies the analytical and numerical treatment of the non-linear problem. Much work has been done in this line, starting with Gunn \& Gott (1972), Gott \& Rees (1975) and Peebles (1980), who studied the spherical collapse in standard CDM (SCDM) models. Lahav et al. (1991), Eke et al. (1996), Brian \& Norman (1998) and others generalized the results to the case of the $\Lambda \mathrm{CDM}$. If the initial density contrast of a spherical perturbation is $\Delta_{i}=1+\delta_{i}$ and its initial radius is $R_{i}$, then the radius of the perturbation $R=r R_{i}$ at later times can be found using the equation:

$$
\frac{\ddot{r}}{r}=-H_{i}^{2}\left[\frac{\Omega_{m, i} \Delta_{i}}{2 r^{3}}+\Omega_{r, i}\left(\frac{a_{i}}{a}\right)^{4}+\frac{(1+3 w) \rho_{D E}}{2 \rho_{c r, i}}\right]
$$

where all quantities with subscript $i$ refer to the initial time. In particular $\Omega_{m, i}$ and $\Omega_{r, i}$ are the density parameters for non- and relativistic matter at that time. After slowing down relative to the scale factor $a(t)$, the perturbation eventually stops at moment $t_{t a}$, when its radius is $R_{t a}$. The radius $R$ formally goes to zero at $\sim 2 t_{t a}$ corresponding 
to redshift $z_{c o l}$. The value of $z_{c o l}$ depends on the amplitude of the initial fluctuation $\delta_{i}$. Instead of $\delta_{i}$ it is however convenient to use the amplitude $\delta_{c}$ as estimated by the linear theory at $z_{c o l}$. For SCDM the value of this density contrast is

$$
\delta_{c}^{*} \simeq 1.68
$$

and does not depend on $z_{c o l}$ (see, e.g., Coles \& Lucchin 1995). For other models $\delta_{c}$ does depend on $z_{c o l}$.

In the contraction stages fluctuations heat up and, unless kinetic energy can be succesfully radiated away, contraction will stop when virial equilibrium is attained and its size is $R_{v}$. Requiring energy conservation and virial equilibrium we obtain the following algebric cubic equation

$$
x^{3}-\frac{1+y\left(a_{t a}\right)}{2 y\left(a_{c o l}\right)} x+\frac{1}{4 y\left(a_{c o l}\right)}=0,
$$

where $x=R_{v} / R_{t a}$ and

$$
y(a)=\frac{1-\Omega_{m}(a)}{\Delta_{i} \Omega_{m}(a)}\left(\frac{R_{t a}}{R_{i}}\right)^{3}\left(\frac{a_{i}}{a}\right)^{3} .
$$

Note that the actual radius of the final virialized halo is often larger than $R_{v}$, owing to deviations from spherical growth in the real world (Macciò, Murante \& Bonometto, 2003). Still, $R_{v}$ is a good starting point for statistical analysis. Multiplying eq. (6) by $2 y$ and taking then $y=0$ (i.e. $\Omega_{m} \equiv 1$ : $\mathrm{SCDM}$ ), we recover that $x=1 / 2$. In general, the root $x$ lays slightly below this value.

Figure 1 shows the linear and non-linear growth of a density contrast, for SCDM, $\Lambda$ CDM and a $\mathrm{RP}$ models normalized to have $z_{c o l}=0$. Similar plots can be made for any redshift of collapse. The Figure can be used to find the initial amplitude $\Delta_{i}$ at any given redshift $z_{i}$ and the value of $\delta_{c}$ for a perturbation collapsing at present. Using the final value of $\Delta$ we obtain the virial density contrast:

$$
\Delta_{c}=\Omega_{m} \Delta .
$$

In the linear and non-linear cases deviations from the SCDM behavior often compensate and the final values of $\delta_{c}$ are just slightly model dependent (see Figure 2 and MMB03 for more details). The spread among the virial density contrasts $\Delta_{c}$, is large as indicated by Figure 3, which shows $\Delta_{c}$ as the function of $\Omega_{m}$ for different models. The

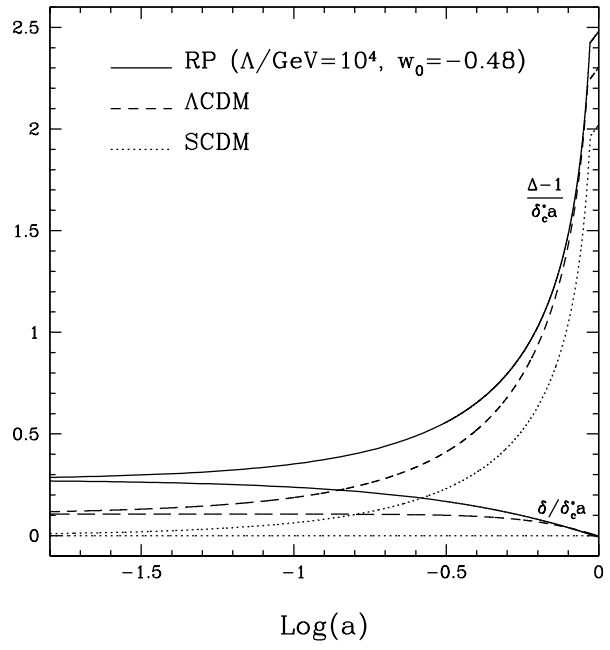

Fig. 1.- Normalized linear (bottom curves) and nonlinear (top curves) amplitude of density fluctuations for SCDM (dotted), $\Lambda \mathrm{CDM}$ (dashed), and RP (full) models. The amplitude of fluctuation was normalized to have collapse if the perturbation at $z_{c o l}=0$. Similar plots can be given for collapse at any other redshift. The density contrast $\Delta=\Delta_{c} / \Omega_{m}$.

evolution of $\Delta_{c}$ with redshift is also very model dependent, as shown by Figure 4 ). We provide an approximation, which is valid at any redshift $z$, provided that we know the matter density parameter $\Omega_{m}$ at that redshift:

$$
\Delta_{c}=178 \Omega_{m}^{\mu\left(\Omega_{m}, \Lambda\right)} .
$$

Here $\mu\left(\Omega_{m}, \Lambda\right)=a+b \Omega_{m}^{c}$ with $c=1(2)$ for RP (SUGRA). Parameters $a$ and $b$ are given by

$$
a=a_{1} \lambda+a_{2}, \quad b=b_{1} \lambda+b_{2},
$$

where

$$
\lambda=\log (\Lambda / \mathrm{GeV})
$$

and the coefficients are given in Table 1 .

Figure 5 shows the dependence on $\lambda$ of the differences $\left|\Delta_{c}^{\text {num }} / \Delta_{c}^{a n}-1\right|$, at $z=0$, for models with $h=0.7$ and different values of $\lambda$, as a function of $\Omega_{m}$. (Here $\Delta_{c}^{\text {num }}$ is obtained from the full numerical treatment, while $\Delta_{c}^{a n}$ is the expression (9)) Discrepancies stay below $0.5 \%$ for any $\Omega_{m} \lesssim 0.15$. However, for large $\lambda$, the approximation is even better: $\lesssim 0.2 \%$, for any $\Omega_{m}$. 


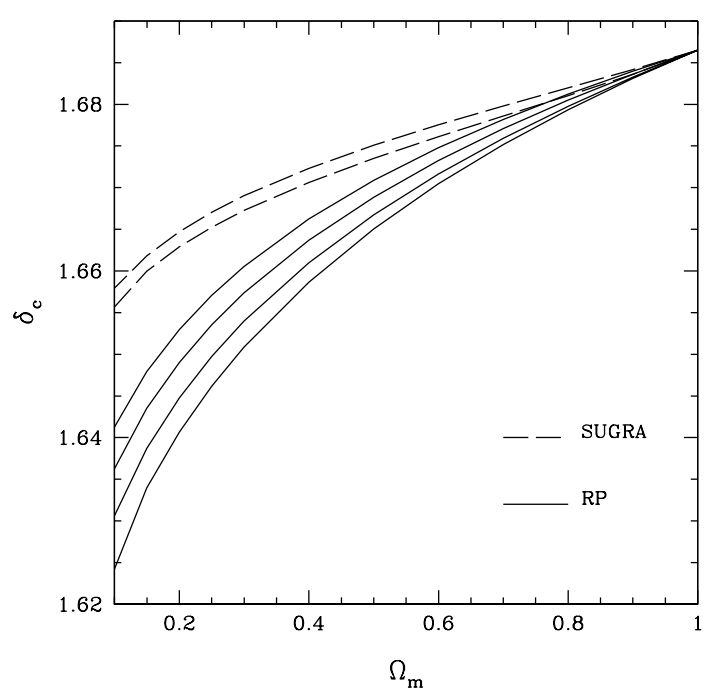

Fig. 2.- The dependence of $\delta_{c}$ on the matter density parameter $\Omega_{m}$ at $z=0$ for $4 \mathrm{RP}\left(\Lambda / \mathrm{GeV}=10^{2}, 10^{4}\right.$, $10^{6}$ and $\left.10^{8}\right)$ and 2 SUGRA models $\left(\Lambda / \mathrm{GeV}=10^{2}\right.$ and $\left.10^{8}\right) . \Lambda$ values increase from top to bottom curves.

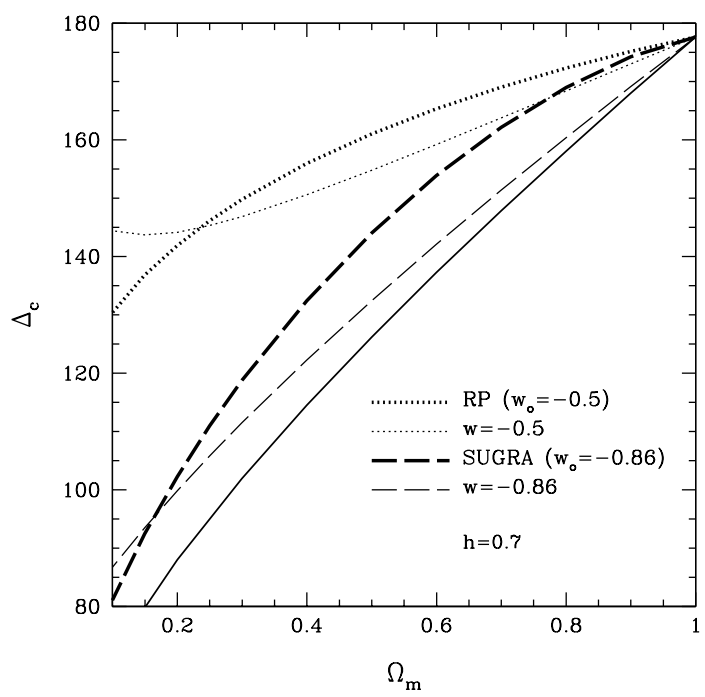

Fig. 3. $-\Omega_{m}$ dependence of $\Delta_{c}$ for different cosmologies. RP and SUGRA models, at $z=0$, have a pressure/density ratio $w_{o}=w$ of the constant $w$ models shown. The full curve is for $\Lambda \mathrm{CDM}$.

\section{The mass function and the linear growth factors for dynamical $\mathrm{DE}$}

We use both the Press-Schechter (PS; 1974) and the Sheth-Tormen (ST; 1999, 2002) approx-

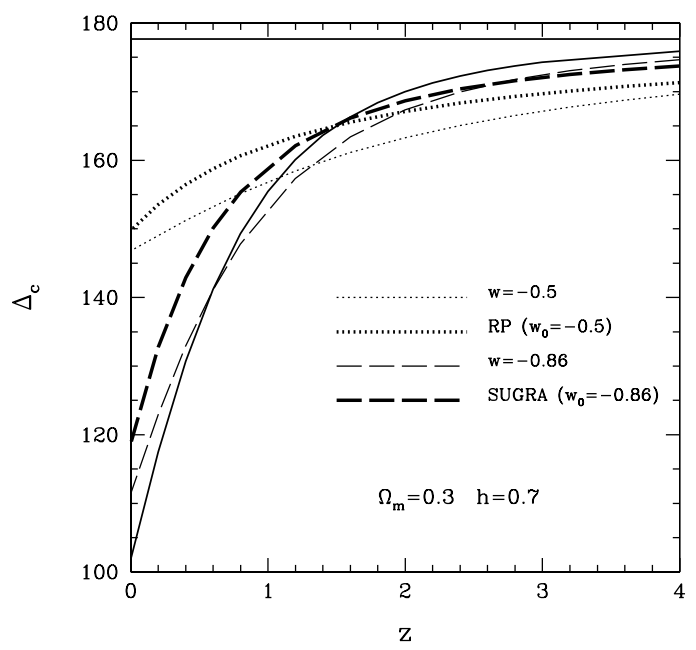

Fig. 4.- Redshift dependence of $\Delta_{c}$ for different cosmologies. RP and SUGRA models, at $z=0$, have a pressure/density ratio $w_{o}=w$ of the constant $w$ models shown. Full curves are for DM and $\Lambda$ CDM.

imations for the mass function of dark matter halos. The value of $\delta_{c}$ defines the bias factor $\nu=\delta_{c} / \sigma_{M}$ for the mass $M$. Here $\sigma_{M}$ is the rms density fluctuation on this scale. The bias factor enters then the expression

$$
f(\nu) \nu d \log \nu=\frac{M}{\rho_{m}} n_{h}(M) M d \log M,
$$

with either

$$
f(\nu) \nu=\sqrt{2 / \pi} \nu \exp \left(-\nu^{2} / 2\right),
$$

or

$$
\begin{aligned}
& f(\nu) \nu= \\
& A\left(1+\nu^{\prime-2 q}\right) \sqrt{2 / \pi} \nu^{\prime} \exp \left(-\nu^{\prime 2} / 2\right),
\end{aligned}
$$

with a small complication in the ST case; here $\nu^{\prime}=\sqrt{a} \nu$ with $a=0.707$, while the constants

Table 1: Interpolation coefficients for $\Delta_{c}$

\begin{tabular}{lllll}
\hline \hline Model & $a_{1}$ & $a_{2}$ & $b_{1}$ & $b_{2}$ \\
\hline RP & $-1.45 \times 10^{-2}$ & 0.186 & -0.011 & 0.22 \\
SUGRA & $-2.25 \times 10^{-3}$ & 0.3545 & -0.01875 & -0.1225 \\
\hline
\end{tabular}




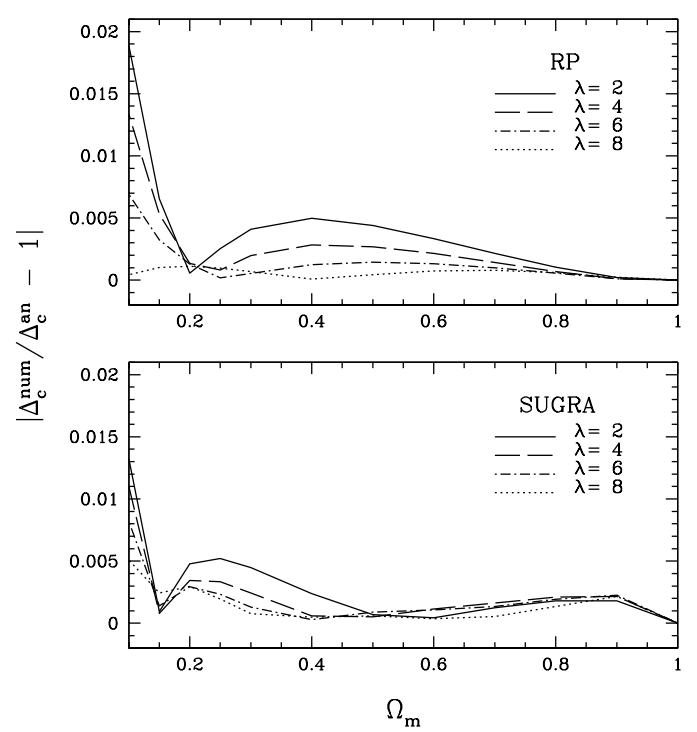

Fig. 5.- Fractional discrepancy between numerical and analytical results on $\Delta_{c}$.

$q=0.3$ and $A=0.3222$. Using eq. (12) we obtain the differential mass function (MF) $n_{h}(M)$ in the PS and ST approximations, once the distribution on bias is given. Here, as usual, we assume a Gaussian $f(\nu)$. Eqs. (12-14) can then be integrated to obtain the halo mass function $n_{h}(>M, z)$ at any redshift $z$.

Such computation must use appropriate values for $\delta_{c}$ and $\sigma_{M}$; the latter are computed by integrating the power spectrum $P(k)$. Its shape depends on specific choice of dynamical DE as our modified CMBFAST program shows. Yet, the dependence is very mild for wavelength smaller than the galaxy cluster scale. On the contrary, as can be seen also from Figure 1, the linear growth factor depends on DE nature in quite a significant way. Figure 6 presents the $z$-dependence of the growth factor for $z$ up to 40 and for a number of different models.

In particular, Figure 6 shows that at redshift $z \geq 2$ the difference between $\Lambda \mathrm{CDM}$ and a model with constant $w=-0.86$ is equal or even smaller than the very difference between this constant- $w$ model and the SUGRA model yielding the same ratio $w$ at $z=0$. However, the latter difference becomes comparable with the former one already at $z \gtrsim 0.5$. A constant- $w$ approximation seems

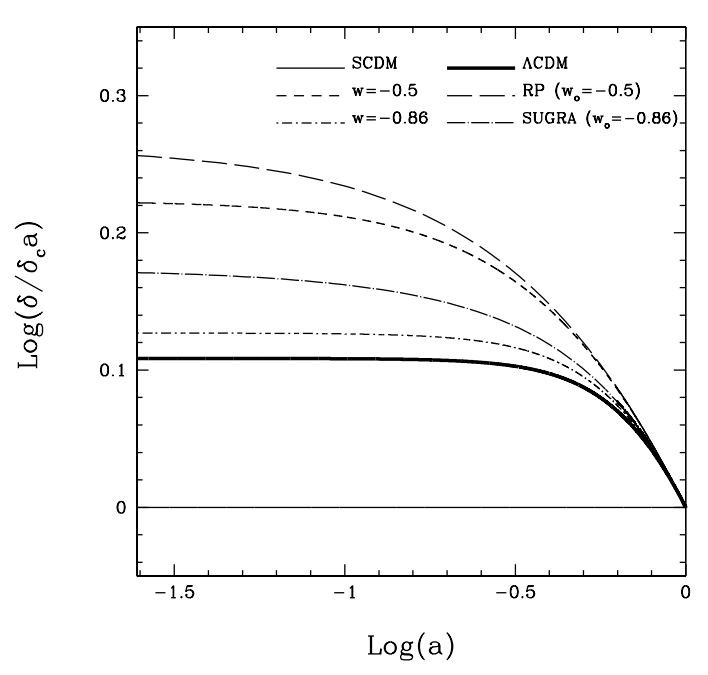

Fig. 6. - Linear growth factor for various models. $w_{o}$ is the value of $w$ at $z=0$.

to perform better for RP models, but this can be mostly ascribed to the fact that the ratio $w$ at $z=0$ is smaller, for these models. Their distance from $\Lambda \mathrm{CDM}$ is therefore greater and the difference between them and constant- $w$ models appears comparatively smaller. However, also in this case, using constant $w$ instead of $\mathrm{RP}$, at $z \geq 4$ is surely misleading.

The linear growth factor shown in Figure 6 is very important for setting initial conditions of $N$-body simulations because linear growth factors are required for the normalization of the power spectrum at the initial redshift $z_{\text {in }}$ of simulations. For these reasons we also give an analytical approximation which reproduces fairly well the behavior of the linear growth factors at $z=40$ for different values of $\Omega_{m}(z=0)$ and $\lambda$ :

$$
\frac{\delta_{c}}{\delta(z=40)}=A+B \lambda+C \lambda^{2} .
$$

The values of the coefficients $A, B, C$ are presented in Table 2 for RP and SUGRA models respectively. At $z=40$, the discrepancies between $\Omega_{m}$ and unity already range around $2-3 \%$. If a simulation must be started at larger $z$, extrapolating the linear growth factor by assuming that $\delta \propto a$ at $z>40$, implies an error smaller than such percentage. This can be still improved by assuming 
that $\delta \propto a^{\Omega_{m}^{q}}$, with $q \simeq 0.4$. The dependence of $q$ on the model and on the energy scale $\lambda$ fixes the second decimal of $q$ and allows a precision better than $0.01 \%$, which is out of the scopes of this analysis.

Mass functions $n(>M, z)$, obtained according to eqs. (12-14), will be compared with simulations in the accompanied paper (Klypin, Macciò, Mainini \& Bonometto 2003). Similar mass functions, obtained from the PS expressions, were used in MMB03 to estimate expected observable differences between models with different dynamical DE.

\section{Evolution of the matter density param- eter}

In RP and SUGRA models, at variance from models with $w=$ const, no analytical expression of $\Omega_{m}(a)$ is readily available. An accurate approximate expression of $\Omega_{m}$, for various redshifts and for different models is useful for various purposes. In particular, it can be used, in conjunction with eqs. (9-10), to find the value of $\Delta_{c}$ at $z \neq 0$.

We found the following fitting formula:

$$
\Omega_{m}(a)=1-\left(1-\Omega_{m, 0}\right) /(1+z)^{\alpha(z, \lambda)},
$$

where $\Omega_{m, 0}$ is the matter density parameter at $z=$ 0 , while $\alpha(z, \lambda)=a+b z^{c}+d /(1+z)$ with $d=0$ for RP models. Parameters $a, b, c$ and $d$ have the same structure as eq. 10. The coefficients are given in Tables 3.

Figure 7 shows the errors of approximation $\left|\Omega_{m}^{\text {num }} / \Omega_{m}^{a n}-1\right|$ as a function of the redshift $z$ for two RP and for two SUGRA models with $\Omega_{m}=0.3$ and $h=0.7$.

Table 2: Coefficients for the linear growth factor

\begin{tabular}{llll}
\hline \hline Parameter & $\Omega_{m}=0.2$ & $\Omega_{m}=0.3$ & $\Omega_{m}=0.4$ \\
\hline SUGRA & & & \\
$A$ & 25.6 & 28.5 & 30.7 \\
$B$ & -0.237 & -0.26 & -0.274 \\
$C$ & 0 & 0 & 0 \\
& & & \\
RP & & & \\
$A$ & 21.3 & 25.1 & 28.2 \\
$B$ & -0.755 & -0.783 & -0.698 \\
$C$ & -0.0125 & -0.0155 & -0.0155 \\
\hline
\end{tabular}

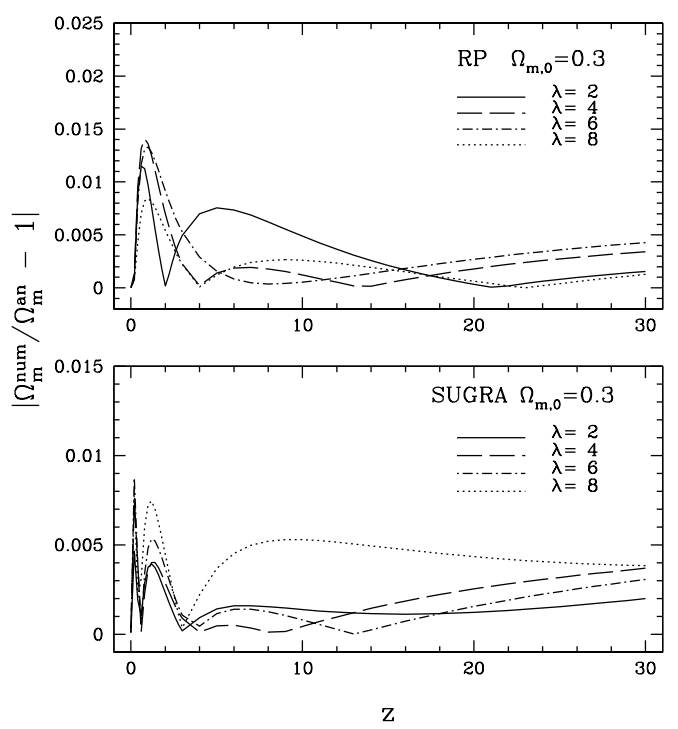

Fig. 7.- Fractional discrepancies between the approximated expression (16) and numerical data.

All that is needed to find the relation between the scale factor $a$ and time in any flat dynamical DE model, is such expression. In fact, let us remind that

$$
\dot{a} / a=H_{o} \sqrt{\rho(a) / \rho_{c r, 0}}
$$

with

$$
\rho(a)=\rho_{m, 0} / a^{3}+\rho_{r} / a^{4}+\dot{\phi}^{2} / 2+V(\phi) .
$$

At low $z$, we can omit the contribution of the radiation density. Therefore, at any time,

$$
\begin{aligned}
\rho_{\phi} & =\frac{\dot{\phi}^{2}}{2}+V(\phi)=\rho_{c r}(a)\left[1-\Omega_{m}(a)\right] \\
& =\rho_{m}(a) \frac{1-\Omega_{m}(a)}{\Omega_{m}(a)}
\end{aligned}
$$

provided that we are dealing with a model, such that the total density is equal to the critical density $\rho_{c r}(a)$. Then, the Friedmann equation reads

$$
\begin{aligned}
\left(\frac{\dot{a}}{a}\right)^{2} & =\frac{8 \pi}{3} G \rho_{m}(a)\left[1+\frac{1-\Omega_{m}(a)}{\Omega_{m}(a)}\right] \\
& =\frac{8 \pi}{3} G \frac{\rho_{m, 0}}{a^{3} \Omega_{m}(a)},
\end{aligned}
$$

so that

$$
\frac{\dot{a}}{a}=H_{o} \sqrt{\frac{\Omega_{m, 0}}{a^{3} \Omega_{m}(a)}} .
$$


TABLE 3

Coefficients For $\Omega_{m}(z)$

\begin{tabular}{llll}
\hline \hline Parameter & \multicolumn{1}{c}{$\Omega_{m}=0.2$} & \multicolumn{1}{c}{$\Omega_{m}=0.3$} & \multicolumn{1}{c}{$\Omega_{m}=0.4$} \\
& & & \\
\hline $\mathrm{RP}$ & & & \\
$a_{1}$ & $-5.638 \times 10^{-3}$ & $-2.119 \times 10^{-2}$ & $-3.365 \times 10^{-2}$ \\
$a_{2}$ & -0.813 & -0.259 & 0.207 \\
$b_{1}$ & $-2.460 \times 10^{-2}$ & $-1.833 \times 10^{-2}$ & $-1.384 \times 10^{-2}$ \\
$b_{2}$ & 1.382 & 0.975 & 0.628 \\
$c_{1}$ & $-5.960 \times 10^{-3}$ & $-6.975 \times 10^{-3}$ & $-8.394 \times 10^{-3}$ \\
$c_{2}$ & $8.460 \times 10^{-2}$ & $9.771 \times 10^{-2}$ & 0.119 \\
& & & \\
SUGRA & & & \\
$a_{1}$ & $-8.466 \times 10^{-3}$ & $-9.161 \times 10^{-3}$ & $-2.035 \times 10^{-2}$ \\
$a_{2}$ & 1.383 & 1.415 & 1.427 \\
$b_{1}$ & $-1.386 \times 10^{-2}$ & $-1.753 \times 10^{-2}$ & $-1.336 \times 10^{-2}$ \\
$b_{2}$ & $-8.521 \times 10^{-3}$ & $-6.890 \times 10^{-3}$ & $-1.289 \times 10^{-2}$ \\
$c_{1}$ & $-3.935 \times 10^{-2}$ & $-4.421 \times 10^{-2}$ & $-4.203 \times 10^{-2}$ \\
$c_{2}$ & 0.710 & 0.688 & 0.682 \\
$d_{1}$ & $2.088 \times 10^{-2}$ & $1.875 \times 10^{-2}$ & $2.212 \times 10^{-2}$ \\
$d_{2}$ & -0.883 & -0.621 & -0.416 \\
\hline
\end{tabular}

This formula is valid regardless of the equation of state of DE. In models with constant $w$, the density $\rho_{D E} \propto a^{-3(1+w)}$ and, therefore, owing to eq. (21),

$$
\Omega_{m}(a)=\left[1+a^{-3 w}\left(\Omega_{m, 0}^{-1}-1\right)\right]^{-1} .
$$

The expressions (16-22), yielding $\Omega_{m}(a)$, as well as eq. (21), yielding $\rho_{c r}$, can be used in $N$-body programs, to determine the trajectories of particles in an expanding universe. In fact, once we know $\Omega_{m}$ and $\rho_{c r}$, we can integrate the Poisson equation $\nabla^{2} \Phi=-4 \pi G a^{2} \rho_{c r} \Omega_{m} \delta_{m}$, yielding the peculiar potential $\Phi$ due to the density fluctuations $\delta_{m}$, obtained from the particle distribution. Then, the equations of motion of each particle

$$
\frac{d \vec{p}}{d a}=-\dot{a} \nabla_{x} \Phi, \quad \frac{d \vec{x}}{d a}=\frac{\vec{p}}{a^{2} \dot{a}}
$$

(see, e.g., Peebles 1980; here $\vec{p} \equiv a \vec{v}$ ) can be integrated, using $\dot{a}$ given by eq. (21), and we obtain the evolution of particle positions, as a function of the scale factor $a$. The $N$-body code ART (Kravtsov et al. 1997), used in the accompanying paper (Klypin, Maccò, Mainini \& Bonometto
2003) to discuss the evolution of models with the dynamical DE and DE with constant $w$, has been modified on these bases.

Figure 8 compares the expansion law $a_{\text {apx }}(t)$, obtained using eqs. (16) and (17), with the numerical behavior $a_{\text {num }}(t)$. Discrepancies seldom exceed $0.4 \%$ and mostly are well below $0.1 \%$. For any practical reasons the errors are negligible.

\section{Discussion}

Observational effects of DE have been considered by various authors, but often models with a constant $w$ are used. Besides of being simpler, constant $w$ models give a feeling that results are generic in a sense that they do not depend on the nature of underlying dark energy. For instance, Wang \& Steinhardt (1998), Steinhardt, Zlatev \& Wang (1999), Zlatev, Wang \& Steinhardt (1999) and Lokas (2002) derived the $\Delta_{c}$ dependence on $\Omega_{m}$ and $w$, in the constant $w$ approximation. Schuecker et al (2003) extended the results to large negative $w$ values to include the case of phantom energy (Caldwell 2002, Schulz \& White 


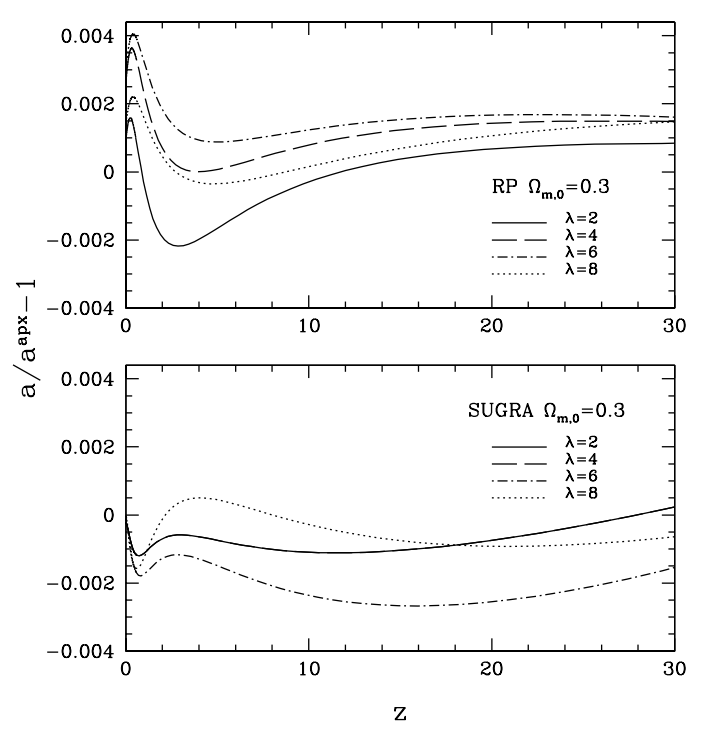

Fig. 8.- Fractional discrepancies between the analytical and numerical integration of eq. (17) to obtain $a(t)$

2001).

Unfortunately, results depend on what is assumed for the DE. Figure 3 shows the dependence of $\Delta_{c}$ on $w$ for models with $\Omega_{m}=0.3$ and $h=0.7$ for three cases: DE is cosmological constant, constant $w \neq-1$, and for dynamical DE with RP or SUGRA potentials. The difference between constant $w$ and dynamical DE is as large as the difference between $\Lambda \mathrm{CDM}$ and a constant $w$. In other words, if we need to consider models more sophisticated than $\Lambda \mathrm{CDM}$, it is not enough to discuss only constant $w \neq-1$. Figure 9 illustrates that the growth factor for dynamical DE cannot be approximated by a model with constant $w$. It seems clear that the Universe "knows" the underlying physics, and predictions depend on the shape of the potential of the scalar field responsible for the DE.

In principle, finding astrophysical quantities which depend on microphysics is far from being unwelcome. Accordingly, the detailed dependence of astrophysical observables on microphysical parameters deserves to be inspected. Let us outline, in particular, that Figure 3 applies to observations at $z=0$, while effects of dynamical DE are also expected at higher $z$. In fact, in Figure 4 we show the $z$ dependence of $\Delta_{c}$, for 3 sets of models char-

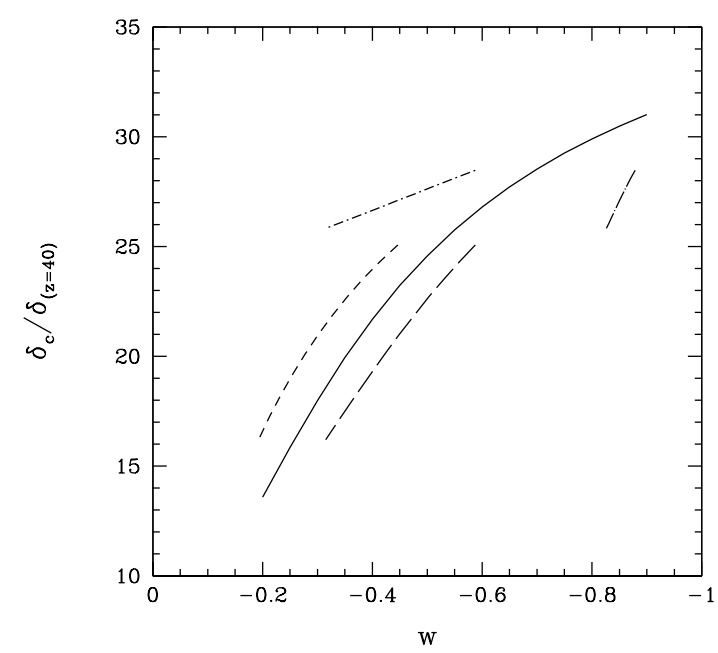

Fig. 9.- The linear growth factor for models with $w=$ const (dotted curve) is compared with linear wrowth factor for RP and SUGRA models (dashed and dot-dashed curves, respectively). RP and SUGRA results are plotted as a function of the value of $w$ they have either at $z=0$ or at $z=40$ (long and short dashes, respectively). The logarithmic energy scale $\lambda$, for both models, ranges here from 2 to 10 . The plot illustrates that the growth factor for dynamical DE cannot be easily approximated by any model with constant $w$.

acterized by the same values of $w$ at $z=0$. The figure shows that the differences between $\Delta_{c}$ 's increase from $z=0$ toward intermediate redshifts, to go back to SCDM values at high redshifts, when ordinary matter gradually approaches critical density. However, at intermediate redshifts, which are most relevant for present and future observations and for the actual dependence on the nature of DE, at these $z$, arises from actual changes of $w$, as shown in Fig. 9. Apart of any consideration concerning fundamental physics, therefore, high- $z \Delta_{c}$ values obtained within constant $w$ approximation risk to create a bias.

Our aim is to facilitate the usage of dynamical DE. We provide the following tools: (i) An approximation for $\Omega_{m}(a)$; (ii) An interpolating expression for $\Delta_{c}$, valid at any redshift for given $\Omega_{m}(a)$; (iii) An analytical expression for the rate of change of the expansion parameter needed for running $N$-body and hydro- simulations; (iv) A 


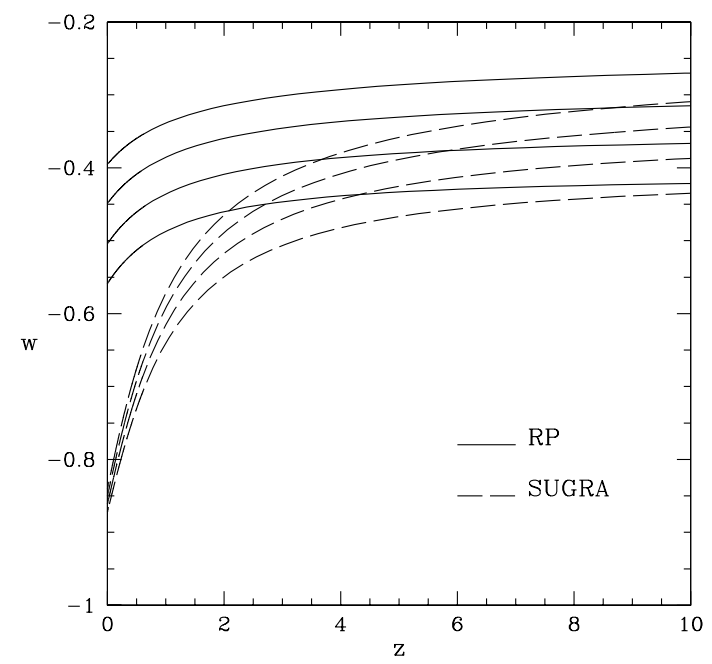

Fig. 10. - Redshift dependence of $w$ for $4 \mathrm{RP}$ and 4 SUGRA models $(\lambda=1,3,5$ and 7$) ; \lambda$ decreases from top to bottom curves.

plot of the linear growth factor, for a number of dynamical DE models, and an analytical approximation for it, to be used to set the initial conditions of $N$-body simulations.

Using these formulae, we modified the ART code, that will be used in the accompanying paper (Klypin, Macciò, Mainini \& Bonometto 2003). In a similar way, other programs dealing with $N$-body interactions or hydrodynamics can be appropriately modified.

\section{REFERENCES}

Balbi, A. et La. 2000, ApJ, 545, L1

Brat, P. \& Martin, J., 1999, Phys.Lett., B468, 40

Brax, P. \& Martin, J., 2000, Phys.Rev. D, 61, 103502

Brax P., Martin J. \& Riazuelo A., 2000, Phys.Rev. D, 62, 103505

Caldwell, R.R., 2002, Phys.Lett.B, 545, 23

Coles, P. \& Lucchin, F., 1995, Cosmology, John Wiley \& Sons.

Dvali, G., Turner, M. 2003, astro-ph/0301510
Efstathiou, G. et al., 2002, MNRAS, 330, 29

Eke, V.R., Cole, S., \& Frenk, C.S., 1996, MNRAS, 282,263

Gott, R. \& Rees, M. 1975, A\&A, 45, 365G

Klypin A., Macciò A.V., Mainini R. \& Bonometto S.A., 2003

Kravtsov A., Klypin A. \& Khokhlov A., 1997 ApJ, $111,73 \mathrm{~K}$

Lahav, O., Lilje, P.R., Primack, J.R. \& Rees, M., 1991, MNRAS, 282, 263

Lacey, C.\& Cole, S., 1993, MNRAS, 262, 627

Lacey, C.\& Cole, S., 1994, MNRAS, 271, 676

Lokas, E.L., 2002, astro-ph/0112031

Macciò A.V., Murante G. \& Bonometto S.A., 2003, astro-ph/0301247, ApJ(May 1 issue)

Mainini R., Macciò A.V. \& Bonometto S.A., 2003 NewA 8, 172

Netterfield, C. B. et al. 2002, ApJ, 571, 604

Peebles P.J.E., 1980, The Large Scale Structure of the Universe, Princeton University Press, Princeton

Percival W.J. et al., 2002, astro-ph/0206256, MNRAS(in press)

Perlmutter S. et al., 1999, ApJ, 517, 565

Pogosian, D., Bond, J.R., \& Contaldi, C. 2003, astro-ph/0301310

Press W.H. \& Schechter P., 1974, ApJ, 187, 425

Ratra B., Peebles P.J.E., 1988, Phys.Rev.D, 37, 3406

Riess, A.G. et al., 1998, AJ, 116, 1009

Schulz A.E. \& White M., 2001 Phys.Rev.D 64, 043514

Schecker P., Caldwell R.R., Boehringer H., Collins C.A. \& Guzzo L., 2003 astro-ph/0211480, A\&A (in press)

Sheth R.K., Mo H.J. \& Tormen G., 2001, MNRAS, 323,1 
Sheth R.K. \& Tormen G., 1999, MNRAS, 308, 119

Sheth R.K. \& Tormen G., 2002, MNRAS, 329, 61

Steinhardt P.J., Zlatev, I. \& Wang L., 1999, Phys Rev D, 59, 123504

Spergel D.N. et al, astro-ph/0302209

Tegmark, M., Zaldarriaga, M., \& Hamilton, A. J. 2001, Phys. Rev. D, 63, 43007

Wang L. \& Steinhardt P.J., 1998, ApJ, 508, 483

Wetterich C., 1988, Nucl.Phys.B, 302, 668

Wetterich C., 1995 A\&A 301, 32

Zlatev, I., Wang L. \& Steinhardt P.J., 1999, Phys Rev Lett, 82, 896

This 2-column preprint was prepared with the AAS IATEX macros v5.0. 